\title{
MRI Assessment of Painful Knee Joint in Meenakshi Mission Hospital and Research Centre, Madurai, Tamil Nadu: A Cross- Sectional Study
}

\author{
A. Sathish ${ }^{1}$, S. Shanmuga Jayanthan ${ }^{2}$, K. Shanthi Priya ${ }^{3}$ \\ ${ }^{1}$ Consultant Radiologist, Department of Radiology and Imaging Science, VIMS Hospital, Salem, Tamil Nadu, ${ }^{2}$ Consultant \\ Radiologist, Department of Radiology and Imaging Sciences, Meenakshi Hospital, Tanjore, Tamil Nadu, ${ }^{3}$ Consultant \\ Radiologist, Medicure Plus Diagnostic Centre, Hyderabad, Telangana, India.
}

Corresponding author: Dr. S. Shanmuga Jayanthan, 27, Third Cross Street, Subbaiya Pillai Nagar, Ammal Sathiram, Karaikal-609604, India

DOI: http://dx.doi.org/10.21276/ijcmsr.2019.4.3.55

How to cite this article: Sathish A, Shanmuga Jayanthan S, Shanthi Priya K. MRI assessment of painful knee joint in meenakshi mission hospital and research centre, Madurai, Tamil Nadu: A cross-sectional study. International Journal of Contemporary Medicine Surgery and Radiology. 2019;4(3):C248-C252.

\section{A B S T R A C T}

Introduction: Knee joint is the most important weight bearing joint capable of performing complex and extensive movements. It is therefore more frequently affected by the traumatic and degenerative conditions. MRI remains the imaging modality of choice for knee joint pathology. It is a non-invasive investigation and provides complete information. Hence, the aim of the present study was to assess the non-osseous knee joint abnormality and to evaluate the incidence of pathology associated with each ligament and meniscus separately.

Material and methods: The present study was a cross-sectional study which was conducted in the Department of Imaging and Interventional Radiology of Meenakshi Mission Hospital and Research Centre, Madurai, Tamil Nadu. It was done among 89 subjects after getting approval from ethical and scientific committee of the hospital from March 2015 to December 2015. The patients who were referred with clinical suspicion of knee joint pathologies and with history of pain in the knee with or without swelling where MRI is used as a modality in diagnosing the cause.

Results: In the present study, joint effusion was present in $83.1 \%$ of the cases and was absent in $16.9 \%$ of the cases. The meniscal type of tear was present clinically in $46.1 \%$ cases and $34.8 \%$ subjects confirmed the meniscal type of tear by MRI. Anterior cruciate ligament tear was seen clinically in $59.6 \%$ cases and $39.3 \%$ through MRI whereas posterior cruciate ligament tear was observed $5.6 \%$ cases clinically and in $5.6 \%$ with the help of MRI. Medial collateral ligament tear was clinically seen in $4.5 \%$ cases and in $12 \%$ cases through MRI whereas lateral collateral ligament tear was observed in $19.1 \%$ cases clinically and in $15.7 \%$ cases through MRI.

Conclusion: Out of the various imaging modalities available to evaluate knee joint, MRI has become the gold standard imaging for evaluating the knee joint pathology. With the use of surface coils it is possible to obtain high resolution images of various ligaments and menisci.

Keywords: MRI Knee Joint, Meniscus Tear, Knee Joint Ligaments, Ligament Tear

\section{INTRODUCTION}

Knee is one of the largest synovial joint in our body. It has three compartments, namely medial tibio-femoral, lateral tibio-femoral and patellofemoral joint. The joint capsule encloses menisci, ligaments and bony articular surfaces. The bony articular surfaces is highly unstable and hence, supporting structures are essential for maintaining joint stability. ${ }^{1}$

The knee is the most important weight bearing joint capable of performing complex and extensive movements. It is therefore more frequently affected by the traumatic and degenerative conditions. Assessment of internal derangements of the knee begins with clinical evaluation including careful physical examination but imaging is fundamental in accurate diagnosis of many of these derangements. Radiographs of the knee are usually the primary imaging studies in knee injury. ${ }^{2}$

In cases of knee joint trauma, clinical examination along with radiographs and even CT scan is not enough to diagnose many internal derangements of this joint. MRI, due to its excellent soft tissue contrast resolution and multi-planar imaging capabilities, provides significant advantages over other imaging techniques in the evaluation of traumatic injuries of knee joint. ${ }^{3}$

MRI remains the modality of choice for imaging knee joint pathology. It is a non-invasive investigation of knee and provides complete information that cannot be obtained by physical examination. MRI demonstrates the sensitivity and specificity of $90 \%$ for detecting meniscal tear and its sensitivity and specificity approaches $100 \%$ in cruciate ligaments injury. ${ }^{4}$ 
The Commonly used sequences for imaging the knee are proton density with fat saturation (PD-FATSAT), fast spin echo (FSE) with fat saturation in axial, sagittal and coronal sequences. Menisci and cruciate ligaments are best imaged in sagittal sequences, whereas, collateral ligaments are best imaged in coronal sequences. ${ }^{5}$

MRI provides excellent soft tissue contrast and is capable of evaluating the soft tissue and bony structures of the knee in multiple planes, which provides significant advantage over conventional arthrography, computed tomography and other imaging techniques. The major application of MRI of knee has been in the evaluation of patient with trauma or suspected internal derangement. ${ }^{6}$

It has become a valuable diagnostic modality for the evaluation of knee joint because it not only depicts osseous lesions, but also provides information on the cartilage, menisci, ligaments and surrounding soft tissues. With recent advances in imaging, it has become possible to evaluate cartilage impairment at an increasingly early stages and even more accurately.

This evolution in imaging methods has advanced alongside the development of new drugs for treating chondral degeneration. However, imaging findings need to be assessed cautiously and always in conjunction with the patient's symptoms, with the aim of avoiding unnecessary treatments. ${ }^{8}$ MRI is considered to be the best non-invasive method for assessing the ligaments, menisci and joint cartilage because of its high soft tissue contrast. It gives information on chondral thickness, morphological abnormalities of the chondral surface, changes in signal within cartilage substances and abnormalities of the subchondral bone. More recent techniques also give information on the biochemical and physiological characteristics of the hyaline cartilage. Thus, MRI usage has been increased in early detection of chondral lesions. ${ }^{9}$

Owing to higher sensitivity and specificity of MRI in the diagnosis of ligament and meniscus pathologies, the aim of the present study is to assess the painful knee joint for the ligamentous and meniscal pathologies with respect to their incidence, types and grade for each of them separately.

\section{MATERIAL AND METHODS}

This was a cross-sectional study conducted in the Department of Imaging and Interventional Radiology of Meenakshi Mission Hospital and Research Centre, Madurai, Tamilnadu among 89 subjects after getting approval from ethical and scientific committee of the hospital from March 2015 to December 2015. The patients who were referred with clinical suspicion of knee joint pathologies with history of pain in the knee with or without swelling where MRI is used as a modality in diagnosing the cause. Also, patients with clinically suspected tears and with restriction of movements at the knee joint following trivial trauma were included in the study. Patients with cardiac pacemakers and metallic implants and who were claustrophobic and were not willing for imaging were completely excluded from this study.

\section{Sample Size and Sample Technique}

According to a study by Nguyen and colleagues, frequent knee pain affects 25\% of adults. (Medscape Education Clinical
Briefs - released 12/20/2011). Based on this prevalence rate and a confidence level of $95 \%$ and power of $90 \%$, the required sample size was calculated using the formula

$\mathrm{n}=\frac{\mathrm{t}^{2} \times \mathrm{p}(1-\mathrm{p})}{\mathrm{m}^{2}}$

$\mathrm{n}=$ required sample size

$\mathrm{t}=$ confidence level at 95\% (standard value of 1.96)

$\mathrm{p}=$ estimated prevalence of knee pain. $=25 \%=0.25$

$\mathrm{m}$ for power of $90 \%=$ margin of error at $10 \%(1-$ alpha $)=0.1$

$$
\begin{aligned}
\text { Sample size } & =\frac{1.96^{2} \times 0.25(1-0.25)}{0.1^{2}} \\
& =\frac{3.8416 \times 0.25 \times 0.75}{0.01}=\frac{0.7203}{0.01}
\end{aligned}
$$

$=72.03$ rounded off to 73 .

Required minimum sample size $=73$

This is calculated for a confidence level of $95 \%$ and power of $90 \%$.

\section{MR imaging protocol}

The images were acquired with the help of PHILIPS MULTIVA 1.5 Tesla MRI scanner. Dedicated surface coil for knee was used. Imaging was done with patient in a supine position with leg by the side in neutral position. The various MR imaging sequences used and their technical parameters are summarised (Figure 1).

After obtaining localiser in all three orthogonal planes, the following sequences are obtained:

Axial T2, Axial STIR

Coronal T1, Coronal STIR

Sagittal STIR

Sagittal PD-FATSAT

After acquisition of images, data collection was done using an observation checklist and data collected was analyzed to achieve the objectives of the study.

\section{STATISTICAL ANALYSIS}

The information collected regarding all the selected cases were recorded in a Master Chart. Data analysis was done with the help of computer using Statistical Package for Social Sciences (SPSS Inc., Chicago, IL, version 22.0 for Windows).

Using this software, frequencies and percentages were calculated for all qualitative variables like sex, history of trauma, mechanism of injury, type of tear etc. Range, mean and standard deviation were calculated for all quantitative variables like age. Student's t- test was used to test the significance of difference for quantitative variables (age). A 'p' value less than 0.05 was taken to denote significant difference.

\section{RESULTS}

In the present study, out of 89 subjects, majority of the subjects belong to $41-50$ years followed by $21-30$ years and 51-60 years. About $69.7 \%$ were males and $30.3 \%$ were females. In the current study, among 89 subjects, about $33.7 \%$ gave history of trauma and $66.3 \%$ gave no history of trauma. Swelling of knee was present in all the 89 (100\%) subjects. 


\begin{tabular}{|l|c|c|c|c|}
\hline & T1 & T2 & STIR & PD-FATSAT \\
\hline TR & 500 & 1000 & 4500 & 2500 \\
\hline TE & 17 & 100 & 30 & 30 \\
\hline Matrix & $320 \times 219$ & $292 \times 232$ & $288 \times 217$ & $288 \times 217$ \\
\hline Slice thickness(mm) & 3 & 4 & 3 & 3.5 \\
\hline Inter-slice spacing (mm) & 1 & 0.5 & 1 & 0.5 \\
\hline FOV & $160 \times 160$ & $160 \times 160$ & 3min 10sec & $2 \mathrm{~min} 45 \mathrm{sec}$ \\
\hline Scan duration & 2min 30sec & 2min 35sec & \multicolumn{2}{|c|}{} \\
\hline \multicolumn{2}{|r|}{ Figure-1: Shows the parameters used for the acquisition of images } \\
\hline
\end{tabular}

\begin{tabular}{|l|c|c|}
\hline \multirow{2}{*}{ Age group } & \multicolumn{2}{|c|}{ Cases } \\
\cline { 2 - 3 } & No. & $\%$ \\
\hline Up to 20 years & 7 & 7.9 \\
\hline $21-30$ years & 16 & 18.0 \\
\hline $31-40$ years & 13 & 14.6 \\
\hline $41-50$ years & 22 & 24.7 \\
\hline $51-60$ years & 16 & 18.0 \\
\hline Above 60 years & 15 & 16.9 \\
\hline Total & 89 & 100 \\
\hline Table-1: Shows the distribution of data based on age-group \\
\multicolumn{2}{|c|}{ distribution among the study subjects } \\
\hline
\end{tabular}

\begin{tabular}{|l|c|c|}
\hline \multirow{2}{*}{ Gender } & \multicolumn{2}{|c|}{ Cases } \\
\cline { 2 - 3 } & 62 & 69.7 \\
\hline Male & 27 & 30.3 \\
\hline Female & 89 & 100 \\
\hline Total & Nable-2: Shows the distribution of data based on gender distri- \\
bution among the study subjects \\
\hline
\end{tabular}

\begin{tabular}{|l|c|c|}
\hline \multirow{2}{*}{ History of Trauma } & \multicolumn{2}{|c|}{ Cases } \\
\cline { 2 - 3 } & 30 & No \\
\hline Yes & 59 & 33.7 \\
\hline No & 89 & 106.3 \\
\hline Total & \multicolumn{2}{|c|}{ Table-3: Shows the distribution of data based on history of } \\
& trauma among the study subjects \\
\hline
\end{tabular}

\begin{tabular}{|l|c|c|}
\hline \multirow{2}{*}{ Knee pain / Swelling } & \multicolumn{2}{|c|}{ Cases } \\
\cline { 2 - 3 } & No & \% \\
\hline Present & 89 & - \\
\hline Absent & - & 100 \\
\hline Total & 89 & 0.0 \\
\hline Table-4: Shows the distribution of data based on knee pain/ \\
swelling among the study subjects \\
\hline
\end{tabular}

\begin{tabular}{|l|c|c|c|c|}
\hline \multirow{2}{*}{ Clinical Suspicion in Knee Joint } & \multicolumn{2}{|c|}{ Present } & \multicolumn{2}{c|}{ Absent } \\
\cline { 2 - 5 } & No. & $\%$ & No. & $\%$ \\
\hline Meniscal Tear & 41 & 46.1 & 48 & 53.9 \\
\hline Anterior cruciate ligament Tear & 53 & 59.6 & 36 & 40.4 \\
\hline $\begin{array}{l}\text { Posterior cruciate ligament } \\
\text { Tear }\end{array}$ & 5 & 5.6 & 84 & 94.4 \\
\hline Medial collateral ligament Tear & 4 & 4.5 & 85 & 95.5 \\
\hline Lateral collateral ligament Tear & 17 & 19.1 & 72 & 80.9 \\
\hline $\begin{array}{l}\text { Table-5: Shows the distribution of data based on clinical suspi- } \\
\text { cion in knee joint among the study subjects }\end{array}$ \\
\hline
\end{tabular}

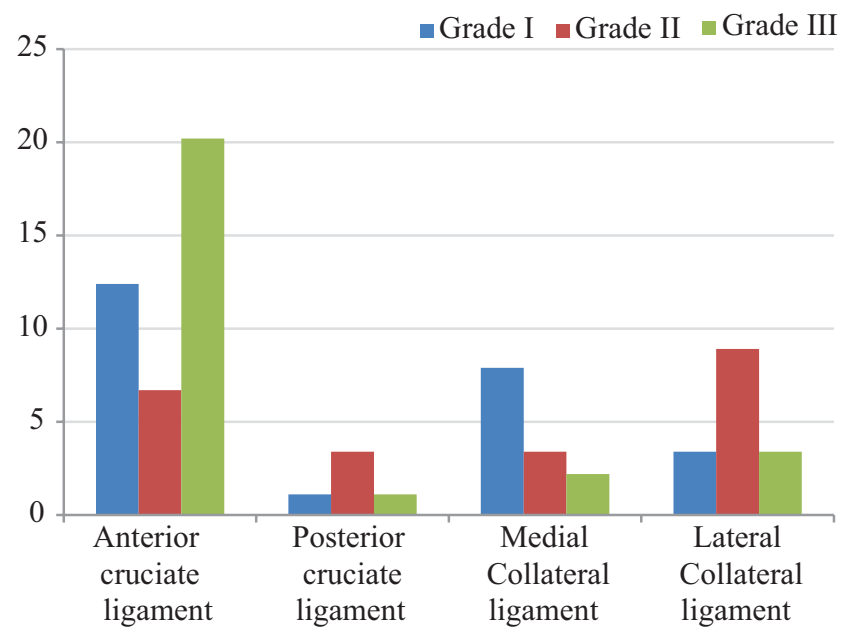

Graph-1: Shows the distribution of data based on type of ligament

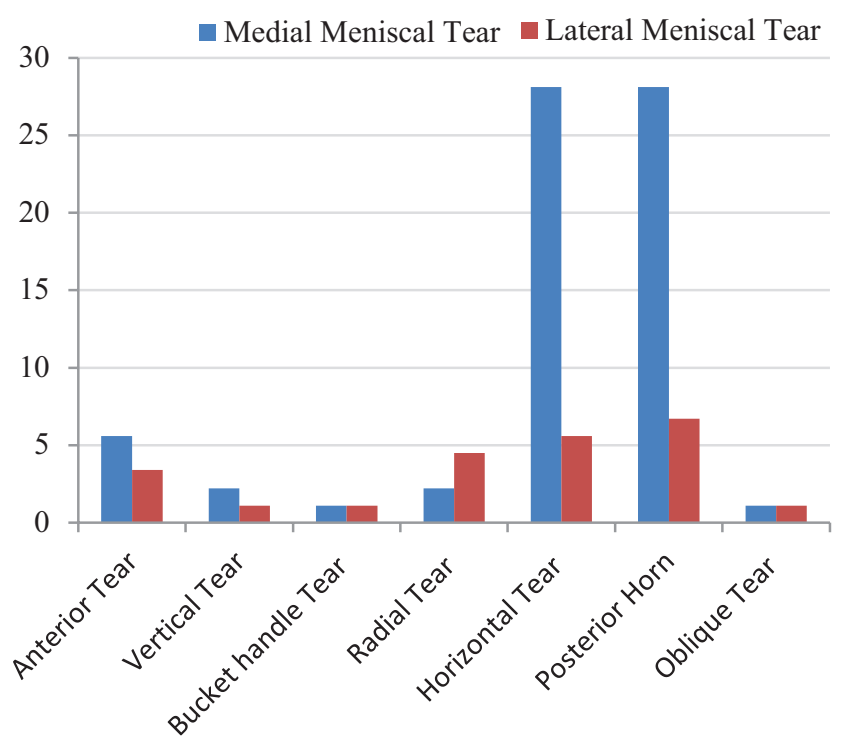

Graph-2: Shows the distribution of data based on type of tear

Meniscal tear was present in $46.1 \%$ of the subjects and absent in $53.9 \%$ of the subjects. Anterior cruciate tear was present in $59.6 \%$ and absent in $40.4 \%$ of the subjects. About $5.5 \%$ posterior cruciate ligament tear was present and $94.4 \%$ was absent among the subjects. Medial collateral ligament tear was present in $4.5 \%$ and was absent in $95.5 \%$ whereas lateral collateral ligament tear was present in $19.1 \%$ and absent in $80.9 \%$ of the subjects (Table 1,2,3,4 and 5).

In the present study, joint effusion was present in $83.1 \%$ of the cases and was not present in $16.9 \%$ of the cases (Table 6). 


\begin{tabular}{|l|c|c|}
\hline \multirow{2}{*}{ Joint effusion } & No. & Cases \\
\cline { 2 - 3 } & 74 & 83.1 \\
\hline Yes & 15 & 16.9 \\
\hline No & 89 & 100 \\
\hline Total & \multicolumn{2}{|c|}{} \\
\hline \multicolumn{2}{|c|}{} \\
\hline
\end{tabular}

\begin{tabular}{|c|c|c|c|c|}
\hline \multirow[t]{3}{*}{ Type of Tear } & \multicolumn{4}{|c|}{ Number of cases present as per } \\
\hline & \multicolumn{2}{|c|}{$\begin{array}{c}\text { Clinical } \\
\text { suspicion }\end{array}$} & \multicolumn{2}{|c|}{ MRI } \\
\hline & No & $\%$ & No & $\%$ \\
\hline Meniscal Tear & 41 & 46.1 & 31 & 34.8 \\
\hline Anterior Cruciate Ligament Tear & 53 & 59.6 & 35 & 39.3 \\
\hline Posterior Cruciate Ligament Tear & 5 & 5.6 & 5 & 5.6 \\
\hline Medial Collateral Ligament Tear & 4 & 4.5 & 12 & 13.5 \\
\hline Lateral Collateral Ligament Tear & 17 & 19.1 & 14 & 15.7 \\
\hline
\end{tabular}

\begin{tabular}{|c|c|c|c|c|c|c|c|c|c|}
\hline \multirow[t]{4}{*}{ Type of Tear } & \multicolumn{8}{|c|}{ No. of cases in } & \multirow[t]{4}{*}{$' p '$} \\
\hline & \multicolumn{4}{|c|}{ Tear's Present cases } & \multicolumn{4}{|c|}{ Tear's Absent cases } & \\
\hline & \multicolumn{2}{|c|}{ Males } & \multicolumn{2}{|c|}{ Females } & \multicolumn{2}{|c|}{ Males } & \multicolumn{2}{|c|}{ Females } & \\
\hline & No & $\%$ & No & $\%$ & No & $\%$ & No & $\%$ & \\
\hline Meniscal Tear & 19 & 61.3 & 12 & 38.7 & 43 & 74.1 & 15 & 25.9 & $\begin{array}{l}0.3105 \\
\text { Not significant }\end{array}$ \\
\hline Anterior Cruciate ligament Tear & 32 & 91.4 & 3 & 8.6 & 30 & 55.6 & 24 & 44.4 & $\begin{array}{l}0.0008 \\
\text { significant }\end{array}$ \\
\hline Posterior Cruciate ligament Tear & 4 & 80 & 1 & 20 & 58 & 69.0 & 26 & 31.0 & $\begin{array}{l}0.5188 \\
\text { Not significant }\end{array}$ \\
\hline Medial Collateral ligament Tear & 10 & 83.3 & 2 & 16.7 & 52 & 67.5 & 25 & 32.5 & $\begin{array}{l}0.2259 \\
\text { Not significant }\end{array}$ \\
\hline Lateral Collateral ligament Tear & 12 & 85.7 & 2 & 14.3 & 50 & 66.7 & 25 & 33.3 & $\begin{array}{l}0.1325 \\
\text { Not significant }\end{array}$ \\
\hline
\end{tabular}

The meniscal type of tear was present clinically $46.1 \%$ and was evaluated through MRI $34.8 \%$ among the study subjects. Anterior cruciate ligament tear was seen clinically $59.6 \%$ and $39.3 \%$ through MRI. Posterior cruciate ligament tear was observed $5.6 \%$ clinically and $5.6 \%$ with the help of MRI. Medial collateral ligament tear was clinically seen in $4.5 \%$ and $12 \%$ through MRI whereas Lateral collateral ligament tear was observed $19.1 \%$ clinically and $15.7 \%$ through MRI (Table 7).

The most common type of tear is the ACL Tear which is frequently seen in male patients which was found to be statistically significant at $\mathrm{p}<0.0008$ (Table 8). In this study, anterior cruciate ligament tear i.e. Grade III (20.2\%) was mostly seen in the subjects followed by Grade I and II. Grade II (3.4\%) was seen maximum in posterior cruciate ligament. Grade I (7.9\%) is observed in medial collateral ligament tear followed by Grade II and Grade III. In lateral collateral ligament, majority of the subjects had Grade II (8.9\%) followed by Grade I and III (Graph no. 1).

It was found that in medial meniscal tear, horizontal tear and posterior horn was seen in majority of the cases (28.1\%) whereas in lateral meniscal tear, posterior horn was the most common site seen among $6.7 \%$ of the subjects. (Graph no. 2).

\section{DISCUSSION}

Imaging of the knee is a great challenge because of its complex anatomy. Various imaging modalities are available for evaluating knee joint abnormalities. Commonly used modalities are conventional radiography, Computed Tomography,MRI and Arthrography.MRI has revolutionised the imaging of knee joint. It is invaluable in examining normal anatomy and diagnosing injuries to the knee. Kinematic MRI has added the advantage in the imaging of knee joint because some diseases are joint position dependent or stress conditions because of its great soft tissue contrast resolution MRI is used as an imaging modality of choice for imaging knee joint pathology. ${ }^{10}$

Some studies have validated the role of MRI in imaging ligamentous injuries. We observed that there are so many advantages of MRI over other imaging modalities during the study period. About 89 patients underwent MRI knee joint, out of which 30 patients had history of trauma. About 82 patients found to have positive findings in imaging to structures like cruciate, collateral ligaments and menisci. ${ }^{11}$

In this study, maximum number of subjects was in the age group of $41-50$ years, which constitute about $24 \%$ of cases. 
About 18\% of cases were in the age group of 21-30 years and 51-60 years. Among the 89 cases studied, 62 were male and 27 were females. It is about $70 \%$ and $30 \%$ respectively. Among the 89 cases studied, 30 had history of trauma and 59 had no history of trauma. It is about $33 \%$ and $66 \%$ respectively and these findings are in concordance with the study done by Haims $\mathrm{AH}$ et al. ${ }^{12}$

Among the subjects, 14 had flexion with varus and internal rotation type of injury, 10 had flexion with valgus and internal rotation type of injury, 6 had flexion with valgus type of injury and 1 patient had pure hyperextension injury. Out of these 14 had presented within one week of injury,10 had presented after one week of injury, 5 after two weeks and 2 patients had presented after three weeks. Among the cases, 41 patients had clinical suspicion of meniscal tear, 53 had ACL tear, 5 had PCL tear, 4 had MCL tear and 17 had LCL tear. Out of 89 cases, 74 had joint effusion which constitutes about $83 \%$. Among the subjects, 18(20.2\%) cases had grade III ACL tear, $6(6.7 \%)$ had grade II tear and $11(12.4 \%)$ had grade I tear and these findings are similar to the study conducted by Recondo JA et al. ${ }^{13}$

In a study conducted by Waleed Hetta et al it was found that among 30 cases, 25 patients had history of trauma,15(60\%) patients had ACL tear, 2(8\%) had PCL tear, 10(40\%) had meniscal tear, 8(32\%) had collateral ligament tear. About 7 patients were with isolated injury, 18 were with combined injuries. ${ }^{14}$

In a study conducted by Arumugam et al, it was found that among 120 patients, 54(45\%) had ACL Tear 15(12.5\%) had PCL tear, 27(22.5\%) had MCL tear, 12(10\%) had LCL tear, $39(32 \%)$ had medial meniscal tear and 27(22\%) had lateral meniscal tear. In another study conducted by Jamal Yaqoob et al it was observed that among 54 patients, 31(57.4\%) had medial meniscal tear, $12(22.2 \%)$ had lateral meniscal tear and 11(20.3\%) had ACL Tear. ${ }^{15,16}$ Hence, further studies with larger sample size are required to agree/ disagree with the results of study and also needs arthroscopic correlation to confirm the MRI findings.

\section{CONCLUSION}

MRI is a valuable imaging modality for evaluation of ligament knee pathologies. Some of the tears involving medial meniscus can be diagnosed confidently with MRI and it scores over arthroscopy.

\section{REFERENCES}

1. Tham SC, Tsou IYY, Chee T SG. Knee and ankle: magnetic resonance imaging findings of normal anatomy and at injury. Ann Acad Med Singapore 2008; 37(1):324-329.

2. Muhle C, Ahn JM, Dieke C. Diagnosis of ACL and meniscal injuries: MR imaging of knee flexion versus extension compared to arthroscopy. Springer Plus 2013; 2(1):213.

3. Chen W-T, Shih TT-F, Tu H-Y, Chen R-C, Shau $\mathrm{W}-\mathrm{Y}$. Partial and complete tear of the anterior cruciate ligament: Direct and indirect MR signs. Acta Radiol 2002; 43(5):511-516.

4. Kostov H, Stojmenki S, Kostova E. reliability assessment of arthroscopic findings versus MRI in ACL injuries of the knee. Acta Inform Med 2014;22(2):111-114.

5. Rodriguez W JR, Vinon EN, Hlems CA, Toth AP. MRI appearance of posterior cruciate ligament tears. AJR Am J Roentgenol 2008; 191(4):W155-W155.

6. Sanders TG, Medynski MA, Feller JF, Lawhorn KW. Bone contusion patterns of the knee at MR imaging: footprint of the mechanism of injury. Radio Graphics 2008; 20(6):S135-S151.

7. Ng WHA, Griffith JF, Hung EHY, Paunipagar B, Law BKY, Yung PSH. Imaging of the anterior cruciate ligament. World J Orthop 2011; 2(8):75-84.

8. Vinson EN, Major NM, Helms CA. The posterolateral corner of the knee. AJR Am J Roentgenol 2008; 190(2):449-458.

9. Chen L, Kim PD, Ahmad CS, Levine WN. Medial collateral ligament injuries of the knee: current treatment concepts. Curr Rev Musculoskeletal Med 2008; 1(5):108-113.

10. Smet AAD. How I diagnose meniscal tears on knee MRI. AJR Am J Roentgenol 2012; 199:481-499.

11. Helms CA. The meniscus: recent advances in MR imaging of the knee. AJR Am J Roentgenol 2002; 179(3):1115-1122.

12. Haims AH, Medvecky MJ, Pavlovich R, Jr, Katz LD.MR imaging of the anatomy of and injuries to the lateral and posterolateral aspects of the knee. AJR Am J Roentgenol 2003; 180(5):647-653.

13. Recondo JA, Salvador E, Villanua JA, Barrera B, Gervas C, Alustiza JM. Lateral stabilizing structures of the knee: functional anatomy and injuries assessed with MR imaging. Radio Graphics 2000; 20(6):S91-S102.

14. Hetta W, Niazi G. MRI in assessment of sports related knee injuries. The Egyptian Journal of Radiology and Nuclear Medicine 2014; 45(3): 1153-1161.

15. Arumugam V, Ganesan GR, Natarajan P. MRI evaluation of acute internal derangement of knee. Open Journal of Radiology 2015; 5(5):66-71.

16. Yaqoob J, Alam MS, Khalid N. Diagnostic accuracy of magnetic resonance imaging in assessment of meniscal and ACL tear: correlation with arthroscopy. Pak J Med Sci 2015; 31(2):263-268.

Source of Support: Nil; Conflict of Interest: None

Submitted: 13-07-2019; Accepted: 20-08-2019; Published online: 23-09-2019 